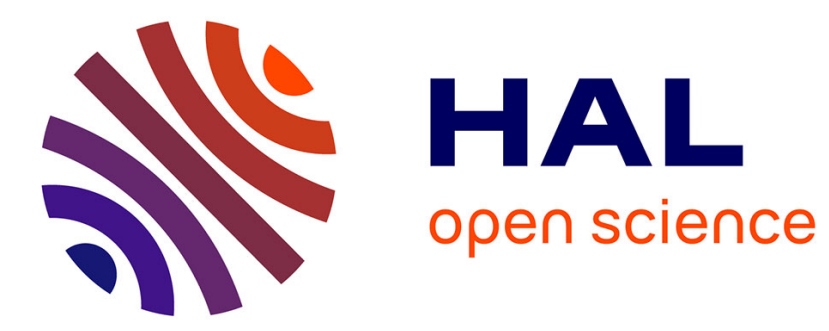

\title{
Metrological Tool for the Characterization of Flame Fronts Based on the Coupling of a Heat Flux Approach with Image Processing Data
}

Steve Rudz, Khaled Chetehouna, Olivier Séro-Guillaume

\section{- To cite this version: \\ Steve Rudz, Khaled Chetehouna, Olivier Séro-Guillaume. Metrological Tool for the Characterization of Flame Fronts Based on the Coupling of a Heat Flux Approach with Image Processing Data. Fire Technology, 2011, 47, pp.491-505. 10.1007/s10694-010-0157-x . hal-00652724}

\section{HAL Id: hal-00652724 \\ https://hal.science/hal-00652724}

Submitted on 16 Dec 2011

HAL is a multi-disciplinary open access archive for the deposit and dissemination of scientific research documents, whether they are published or not. The documents may come from teaching and research institutions in France or abroad, or from public or private research centers.
L'archive ouverte pluridisciplinaire HAL, est destinée au dépôt et à la diffusion de documents scientifiques de niveau recherche, publiés ou non, émanant des établissements d'enseignement et de recherche français ou étrangers, des laboratoires publics ou privés. 


\title{
Metrological tool for the characterization of flame fronts based on the coupling of heat flux approach with image processing data
}

\author{
S. Rudz ${ }^{1,2}$, K. Chetehouna ${ }^{2}$, O. Séro-Guillaume ${ }^{1}$ \\ ${ }^{1}$ LEMTA (UMR 7563 CNRS/INPL/UHP) 2, avenue de la Forêt de Haye - 54504 \\ Vandoeuvre les Nancy cedex. \\ ${ }^{2}$ ENSI de Bourges, Institut PRISME UPRES EA 4229 EP-RES, 88 bd Lahitolle, \\ 18020 Bourges cedex, France. \\ E-mail: khaled.chetehouna@ensi-bourges.fr \\ Phone: +33 248484065 \\ Fax: $\quad+33248484050$
}

\begin{abstract}
The aim of this paper is to present some improvements in the metrology of forest fire flames by coupling image processing and radiative heat flux measurement. A new metrological tool using a visual video camera and a specific multiple thermal sensor is proposed. By means of an appropriate segmentation algorithm and the Direct Linear Transformation, the image processing methodology gives the forward or the backward fire front positions as input data to a radiative heat flux approach. Using a simplified flame model, this technique provides fire front positions versus time, and average values for the flame length, flame depth, flame tilt angle, apparent flame temperature and flame emissivity. The rates of spread, obtained by a linear regression of the determined fire front positions, as well as the thermal and radiative properties of the flame, are compared favorably to those given in the literature.
\end{abstract}

Keywords: Simplified flame model, radiative thermal methodology, multiple thermal sensors, inverse method, image processing 


\section{Nomenclature}

$y_{f}^{B}[\mathrm{~m}]$

$y_{f}^{F}[\mathrm{~m}]$

$l_{f}[\mathrm{~cm}]$

$T_{f}[\mathrm{~K}]$

$K_{f}\left[\mathrm{~m}^{-1}\right]$

$B\left[\mathrm{~W} \cdot \mathrm{m}^{-2} \cdot \mathrm{K}^{-4}\right]$

$W[\mathrm{~m}]$

$C_{c o}[\mathrm{~J} / \mathrm{K}]$

$\hat{R}[\mathrm{~K} / \mathrm{W}], R_{e_{1}}[\mathrm{~K} / \mathrm{W}]$

$\delta_{f}[\mathrm{~cm}]$

Greek symbols

$\left.\alpha_{s}{ }^{\circ}\right]$

$\alpha_{f}\left[{ }^{\circ}\right]$

$\alpha_{w}\left[{ }^{\circ}\right]$

$\Phi_{i}^{t h}[W]$

$\Sigma_{f}$

$\xi$

$\varphi_{i}^{\exp }[\mathrm{W}]$

$\theta_{c o_{i}}[\mathrm{~K}]$

$\theta_{s t}[\mathrm{~K}]$

$\Phi_{i}^{\exp }[W]$

$\varepsilon_{f}$ backward fire front position

forward fire front position

flame length

apparent flame temperature

flame extinction coefficient

Stefan-Boltzmann constant

half-width of the flame

copper plate capacity

thermal resistances

mean flame thickness

slope angle of combustion table from the horizontal plane

flame tilt angle

flame inclination angle due to wind

theoretical radiative heat flux received by the $i^{\text {th }}$ target of sensor

horizontal plane of the burning area

decision variables vector

experimental heat flux received by the $i^{\text {th }}$ target of sensor

temperature of $i^{\text {th }}$ copper plate

steel frame temperature

experimental radiative heat flux received by the $i^{\text {th }}$

target of sensor

flame emissivity 


\section{Introduction}

In wildland fires the most advanced line of the fire, orthogonal to the propagation direction, defines the so-called fire front position. From this position the rate of spread, which is an important parameter in fire risk management, can be easily deduced. In several laboratory experiments and controlled fires [1, 2, 3, 4] various devices and protocols have been used to detect the fire front position. The following tools are widely used in fire metrology: threads positioned perpendicular to the propagation direction, thermocouples aligned on the fire spreading axis and deployment of wireless sensor networks on the observed field [5]. The drawback with these devices, however, is that they give a discontinuous signal and a limited amount of data. More recently, image processing methods have been used, enabling the front position to be extracted from video (or infrared) recordings by different types of image processing algorithms $[6,7,8]$. These methods are simple, cheap, easy to use and give positions all along the fire front contour, unlike threads or thermocouples which only give mean positions. Concurrently, a physical measurement tool has been designed [9] to access both average geometrical (length, tilt angle, depth) and physical (temperature, radiative coefficients) flame characteristics by means of the inverse method and a simplified flame model. This model has been derived using the hypothesis of a thin flame $[4,10]$ in order to avoid integration of the Radiative Transfer Equation. In the expression of heat fluxes, the above-mentioned parameters are involved and it is relevant to determine these parameters by minimizing the differences (in the Euclidean norm) between calculated and computed heat fluxes during propagation. Unfortunately, the fire front positions are parameters of the radiative heat flux method. This is a weakness of the method because if too many positions are to be identified, the number of variables in the optimisation process increases the difficulty of finding a minimum. Therefore, the key point to improve the radiation heat transfer approach is to reduce the number of parameters. Recently [11], image processing and radiation heat transfer measurement methods have been favourably compared and both give the same positions for the fire front.

The aim of this paper is to combine these two approaches in order to reduce the number of parameters by inputting the fire front positions given by image 
processing into the radiation heat transfer measurement method. The second section summarizes the formulae required for the optimisation protocol and describes the wireless multiple thermal sensor used. The image processing approach is detailed in section three. In the fourth section, we present the objective function and the optimisation algorithm. The fifth section describes the experimental set-up. The paper concludes with the presentation of some results and discussions.

\section{Radiative heat fluxes}

\subsection{Theoretical modelling}

In this section we derive the equations necessary to calculate the theoretical radiative heat flux emitted by the flame and received by a wireless thermal sensor [9]. The sensor is centred at the end of the combustion table at a point $\mathbf{M}\left(x_{0}, y_{0}\right)$ on top of the vegetation bed. The inclination angle of the combustion table from the horizontal plane is $\alpha_{s}$. The flame model proposed in this paper is derived from the radiative transfer equation [12] as explained below. In the model, the thermal effects of the surrounding walls are considered negligible as the walls are covered by a thermal insulator, and the flame is considered as a parallelepiped with variable backward and forward positions respectively $y_{f}^{B}$ and $y_{f}^{F}$ (see Figure 1 ), characterized by the following average parameters: length $l_{f}$, tilt angle $\alpha_{f}$, temperature $T_{f}$ and an extinction coefficient $K_{f}$. 


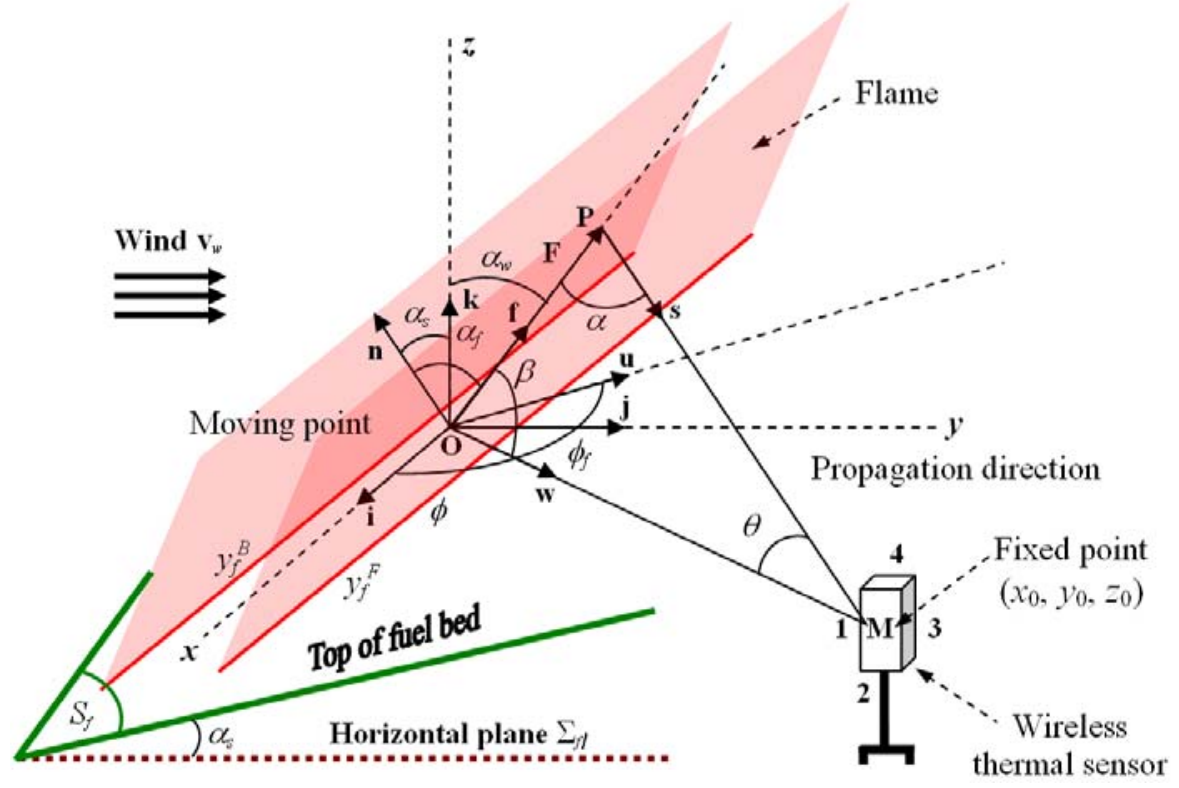

Figure 1. Schematic view of flame and heat flux sensor.

The theoretical radiative heat flux $\Phi_{i}^{\text {th }}$ coming from the fire front and received by a target with a unit vector $\mathbf{n}_{\mathbf{i}}$ can be written $[4,10]$ :

$\Phi_{i}^{t h}(\mathbf{M})=\frac{B}{\pi} \int_{\Sigma_{f}} K_{f} T_{f}^{4} d x_{f} d y_{f} \frac{\cos \alpha_{f}}{\cos \alpha_{s}} \frac{[\cos \beta-\cos (\theta+\beta)] \mathbf{w} \cdot \mathbf{n}_{\mathbf{i}}-(1-\cos \theta) \mathbf{f} \cdot \mathbf{n}_{\mathbf{i}}}{r \sin ^{2} \beta}$

The convective heat flux is the same on each face given the small size of the gauges. In this study the rear gauge never sees the fire front; it only measures the convective heat flux contribution. The rear gauge measurements are therefore subtracted from the others in order to obtain only the radiative contribution. In this case, the unit vector $\mathbf{n}_{\mathbf{i}}$ has two directions for the wireless thermal sensor: $\mathbf{n}_{\mathbf{i}}=\mathbf{j}$ (for the gauge facing the fire) and $\mathbf{n}_{\mathbf{i}}=\mathbf{i}$ or $\mathbf{n}_{\mathbf{i}}=-\mathbf{i}$ (for gauges 2 and 4 oriented obliquely to the flames and perpendicular to the gauge facing the fire). Let us now consider these two configurations:

Gauge facing the fire $\left(\mathbf{n}_{\mathbf{i}}=\mathbf{j}\right)$.

In this case we must calculate:

$\Phi_{a}^{t h}(\mathbf{M})=\frac{B}{\pi} \int_{\Sigma_{f}} K_{f} T_{f}^{4} d x_{f} d y_{f} \frac{\cos \alpha_{f}}{\cos \alpha_{s}} \frac{[\cos \beta-\cos (\theta+\beta)] \mathbf{w} \cdot \mathbf{j}-(1-\cos \theta) \mathbf{f} \cdot \mathbf{j}}{r \sin ^{2} \beta}$ 
Note that $\mathbf{w} \cdot \mathbf{j}=\sin \phi$ and $\mathbf{f} \cdot \mathbf{j}=\sin \alpha_{w} \sin \phi_{f}$, so the theoretical heat flux received by the front face of the wireless thermal sensor can be written as:

$$
\begin{aligned}
\Phi_{a}^{\text {th }}(\mathbf{M})= & \frac{B}{\pi} \int_{\Sigma_{f}} K_{f} T_{f}^{4} d x_{f} d y_{f} \frac{\cos \alpha_{f}}{r \sin ^{2} \beta \cos \alpha_{s}} \times \\
& {\left[\left(\sin \phi \cos \beta-\sin \alpha_{w} \sin \phi_{f}\right)(1-\cos \theta)+\sin \phi \sin \beta \sin \theta\right] }
\end{aligned}
$$

where $\alpha_{w}=(\mathbf{k}, \mathbf{f})$ is the flame inclination angle due to the competition between buoyancy and wind.

Gauges oriented obliquely to the flames (gauge 4) $\left(\mathbf{n}_{\mathbf{i}}=\mathbf{i}\right)$.

We must calculate:

$\Phi_{4}^{t h}(\mathbf{M})=\frac{B}{\pi} \int_{\Sigma_{f}^{(4)}} K_{f} T_{f}^{4} d x_{f} d y_{f} \frac{\cos \alpha_{f}}{\cos \alpha_{s}} \frac{[\cos \beta-\cos (\theta+\beta)] \mathbf{w} \cdot \mathbf{i}-(1-\cos \theta) \mathbf{f} \cdot \mathbf{i}}{r \sin ^{2} \beta}$

Inserting $\mathbf{w} \cdot \mathbf{i}=\cos \phi$ and $\mathbf{f} \cdot \mathbf{i}=\sin \alpha_{w} \cos \phi_{f}$ in this equation, we obtain:

$$
\begin{aligned}
\Phi_{4}^{\text {th }}(\mathbf{M})= & \frac{B}{\pi} \int_{\Sigma_{f}^{(4)}} K_{f} T_{f}^{4} d x_{f} d y_{f} \frac{\cos \alpha_{f}}{r \sin ^{2} \beta \cos \alpha_{s}} \times \\
& {\left[\left(\cos \phi \cos \beta-\sin \alpha_{w} \cos \phi_{f}\right)(1-\cos \theta)+\cos \phi \sin \beta \sin \theta\right] }
\end{aligned}
$$

where $\Sigma_{f}^{(4)}$ is the part of $\Sigma_{f}$ viewed by gauge 4 of the wireless thermal sensor. Gauges oriented obliquely to the flames (gauge 2) $\left(\mathbf{n}_{\mathbf{i}}=-\mathbf{i}\right)$.

We must calculate:

$$
\Phi_{2}^{t h}(\mathbf{M})=\frac{B}{\pi} \int_{\Sigma_{f}^{(2)}} K_{f} T_{f}^{4} d x_{f} d y_{f} \frac{\cos \alpha_{f}}{\cos \alpha_{s}} \frac{(1-\cos \theta) \mathbf{f} \cdot \mathbf{i}-[\cos \beta-\cos (\theta+\beta)] \mathbf{w} \cdot \mathbf{i}}{r \sin ^{2} \beta}
$$

Inserting $\mathbf{w} \cdot \mathbf{i}=\cos \phi$ and $\mathbf{f} \cdot \mathbf{i}=\sin \alpha_{w} \cos \phi_{f}$ in this equation, we obtain:

$$
\begin{aligned}
\Phi_{2}^{t h}(\mathbf{M})= & -\frac{B}{\pi} \int_{\Sigma_{f}^{(2)}} K_{f} T_{f}^{4} d x_{f} d y_{f} \frac{\cos \alpha_{f}}{r \sin ^{2} \beta \cos \alpha_{s}} \times \\
& {\left[\left(\cos \phi \cos \beta-\sin \alpha_{w} \cos \phi_{f}\right)(1-\cos \theta)+\cos \phi \sin \beta \sin \theta\right] }
\end{aligned}
$$


where $\Sigma_{f}^{(2)}$ is the part of $\Sigma_{f}$ viewed by gauge 2 of the wireless thermal sensor, $\cos \beta=\sin \alpha_{w} \cos \left(\phi-\phi_{f}\right), \quad \sin ^{2} \beta=1-\sin ^{2} \alpha_{w} \cos ^{2}\left(\phi-\phi_{f}\right) \quad$ and $\cot \theta=\frac{r}{l_{f} \sin \beta}-\cot \beta$.

In this study, the flame propagates in the direction of the target, meaning that the projection $\mathbf{u}$ of the unit flame vector $\mathbf{f}$ on the plane $\Sigma_{f}$ is collinear to the unit vector $\mathbf{w}$. Consequently, the angle $\phi=\phi_{f}$ and the flame angles are related by $\alpha_{f}=\alpha_{w}+\alpha_{s}$. So, we have $\cos \beta=\sin \alpha_{w}, \quad \sin \beta=\cos \alpha_{w} \quad$ and $\cot \theta=\frac{r}{l_{f} \cos \alpha_{w}}-\tan \alpha_{w}$. Moreover, we can see that $\sin \theta=\frac{l_{f} \cos \alpha_{w}}{\sqrt{r^{2}-2 l_{f} r \sin \alpha_{w}+l_{f}^{2}}}, \quad \cos \theta=\frac{r-l_{f} \sin \alpha_{w}}{\sqrt{r^{2}-2 l_{f} r \sin \alpha_{w}+l_{f}^{2}}}, \quad \sin \phi=\frac{Y}{r}$, $\cos \phi=\frac{X}{r}$; so, we can write:

$\Phi_{a}^{t h}(\mathbf{M})=\frac{B}{\pi} \int_{x_{0}+W}^{x_{0}-W} d X \int_{y_{0}-y_{f}^{B}}^{y_{0}-y_{f}^{F}} K_{f} T_{f}^{4} \frac{\cos \left(\alpha_{w}+\alpha_{s}\right)}{\cos \alpha_{s}} \frac{Y l_{f}}{r^{2} \sqrt{r^{2}-2 l_{f} r \sin \alpha_{w}+l_{f}^{2}}} d Y$

$\Phi_{4}^{t h}(\mathbf{M})=\frac{B}{\pi} \int_{y_{0}-y_{f}^{B}}^{y_{0}-y_{f}^{F}} d Y \int_{x_{0}+W}^{x_{0}} K_{f} T_{f}^{4} \frac{\cos \left(\alpha_{w}+\alpha_{s}\right)}{\cos \alpha_{s}} \frac{X l_{f}}{r^{2} \sqrt{r^{2}-2 l_{f} r \sin \alpha_{w}+l_{f}^{2}}} d X$

$\Phi_{2}^{t h}(\mathbf{M})=-\frac{B}{\pi} \int_{y_{0}-y_{f}^{B}}^{y_{0}-y_{f}^{F}} d Y \int_{x_{0}}^{x_{0}-W} K_{f} T_{f}^{4} \frac{\cos \left(\alpha_{w}+\alpha_{s}\right)}{\cos \alpha_{s}} \frac{X l_{f}}{r^{2} \sqrt{r^{2}-2 l_{f} r \sin \alpha_{w}+l_{f}^{2}}} d X$

where $r=\sqrt{X^{2}+Y^{2}}, X=x_{0}-x_{f}, Y=y_{0}-y_{f}$ and $\left(x_{0}, y_{0}\right)$ is the location on the plane $\Sigma_{f}$ of a target measuring the radiative heat flux coming from the flame. $W$ is the half-width of the flame and $x_{f}, y_{f}$ are the fire coordinates on the x-axis and $\mathrm{y}$-axis respectively.

By integrating equation (8) over the $y$-axis and equations (9) and (10) over the $x$ axis, the theoretical radiative heat fluxes received by the multiple thermal gauges become: 


$$
\begin{aligned}
& \Phi_{a}^{t h}(\mathbf{M})=\frac{B}{\pi} \int_{x_{0}+W}^{x_{0}-W} K_{f} T_{f}^{4} \frac{\cos \left(\alpha_{w}+\alpha_{s}\right)}{\cos \alpha_{s}} \times \\
& \ln \left(\frac{R_{F}(X)}{R_{B}(X)} \frac{l_{f}-R_{B}(X) \sin \alpha_{w}+\sqrt{\left(R_{B}(X)-l_{f} \sin \alpha_{w}\right)^{2}+l_{f}^{2} \cos ^{2} \alpha_{w}}}{l_{f}-R_{F}(X) \sin \alpha_{w}+\sqrt{\left(R_{F}(X)-l_{f} \sin \alpha_{w}\right)^{2}+l_{f}^{2} \cos ^{2} \alpha_{w}}}\right) d X \\
& \Phi_{4}^{t h}(\mathbf{M})=\frac{B}{\pi} \int_{y_{0}-y_{f}^{B}}^{y_{0}-y_{f}^{F}} K_{f} T_{f}^{4} \frac{\cos \left(\alpha_{w}+\alpha_{s}\right)}{\cos \alpha_{s}} \times \\
& \ln \left(\frac{\bar{R}_{2}(Y)}{\bar{R}_{1}(Y)} \frac{l_{f}-\bar{R}_{1}(Y) \sin \alpha_{w}+\sqrt{\left(\bar{R}_{1}(Y)-l_{f} \sin \alpha_{w}\right)^{2}+l_{f}^{2} \cos ^{2} \alpha_{w}}}{l_{f}-\bar{R}_{2}(Y) \sin \alpha_{w}+\sqrt{\left(\bar{R}_{2}(Y)-l_{f} \sin \alpha_{w}\right)^{2}+l_{f}^{2} \cos ^{2} \alpha_{w}}}\right) d Y \\
& \Phi_{2}^{t h}(\mathbf{M})=-\frac{B}{\pi} \int_{y_{0}-y_{f}^{B}}^{y_{0}-y_{f}^{F}} K_{f} T_{f}^{4} \frac{\cos \left(\alpha_{w}+\alpha_{s}\right)}{\cos \alpha_{s}} \times \\
& \ln \left(\frac{\tilde{R}_{2}(Y)}{\tilde{R}_{1}(Y)} \frac{l_{f}-\tilde{R}_{1}(Y) \sin \alpha_{w}+\sqrt{\left(\tilde{R}_{1}(Y)-l_{f} \sin \alpha_{w}\right)^{2}+l_{f}^{2} \cos ^{2} \alpha_{w}}}{l_{f}-\tilde{R}_{2}(Y) \sin \alpha_{w}+\sqrt{\left(\tilde{R}_{2}(Y)-l_{f} \sin \alpha_{w}\right)^{2}+l_{f}^{2} \cos ^{2} \alpha_{w}}}\right) d Y \\
& R_{B}(X)=\sqrt{\left(y_{0}-y_{f}^{B}\right)^{2}+X^{2}}, \quad R_{F}(X)=\sqrt{\left(y_{0}-y_{f}^{F}\right)^{2}+X^{2}}, \\
& \bar{R}_{1}(Y)=\sqrt{Y^{2}+\left(x_{0}+W\right)^{2}}, \quad \bar{R}_{2}(Y)=\sqrt{Y^{2}+x_{0}^{2}}, \quad \tilde{R}_{1}(Y)=\sqrt{Y^{2}+x_{0}^{2}} \quad \text { and } \\
& \tilde{R}_{2}(Y)=\sqrt{Y^{2}+\left(x_{0}-W\right)^{2}} \text {. }
\end{aligned}
$$

In this work the fire propagated through a fuel bed under slope and no-wind conditions, and the heat flux sensor was centred on the $x$-axis on $x_{0}=0$. According to this experimental configuration, $\Phi_{l}^{t h}(\mathbf{M})=\Phi_{2}^{t h}(\mathbf{M})=\Phi_{4}^{t h}(\mathbf{M})$ and equations (11) to (13) can be expressed as:

$$
\begin{aligned}
& \Phi_{a}^{t h}(\mathbf{M})=\frac{B}{\pi} \int_{W}^{-W} K_{f} T_{f}^{4} \ln \left(\frac{\sqrt{\left(y_{0}-y_{f}^{F}\right)^{2}+X^{2}}}{\sqrt{\left(y_{0}-y_{f}^{B}\right)^{2}+X^{2}}} \frac{l_{f}+\sqrt{\left(y_{0}-y_{f}^{B}\right)^{2}+X^{2}+l_{f}^{2}}}{l_{f}+\sqrt{\left(y_{0}-y_{f}^{F}\right)^{2}+X^{2}+l_{f}^{2}}}\right) d X \\
& \Phi_{l}^{t h}(\mathbf{M})=\frac{B}{\pi} \int_{y_{0}-y_{f}^{B}}^{y_{0}-y_{f}^{F}} K_{f} T_{f}^{4} \ln \left(\frac{Y}{\sqrt{Y^{2}+W^{2}}} \frac{l_{f}+\sqrt{Y^{2}+W^{2}+l_{f}^{2}}}{l_{f}+\sqrt{Y^{2}+l_{f}^{2}}}\right) d Y
\end{aligned}
$$




\subsection{Experimental determination}

The wireless heat flux sensor used in this paper consists of a steel body, 4 copper plates, a thin heat insulating layer, a glue layer and 9 type $\mathrm{K}$ thermocouples (see Figure 2). From a previous theoretical study conducted by Chetehouna et al. [9], the total heat flux received by each gauge is calculated as follows:

$$
\varphi_{i}^{\exp }(t)=C_{c o} \frac{d \theta_{c o_{i}}}{d t}+\left(\frac{1}{\hat{R}}+\frac{1}{R_{e_{1}}}\right) \theta_{c o_{i}}-\frac{1}{\hat{R}} \theta_{s t}
$$

where $\varphi_{i}^{\exp }(i=1,2,4)$ is the total heat flux received by the $i^{\text {th }}$ face of the multigauge heat flux sensor, $\theta_{c o_{i}}$ is the $\mathrm{i}^{\text {th }}$ copper plate temperature and $\theta_{s t}$ is the sensor body temperature. $C_{c o}$ is the specific heat capacity of each gauge, $\hat{R}$ is a thermal resistance modelling the contact between the copper plate and the steel body and $R_{e_{1}}$ models the heat transfer between the sensor and the surroundings. These physical parameters included in (16) were all obtained by calibration experiments and are provided in Table 1.

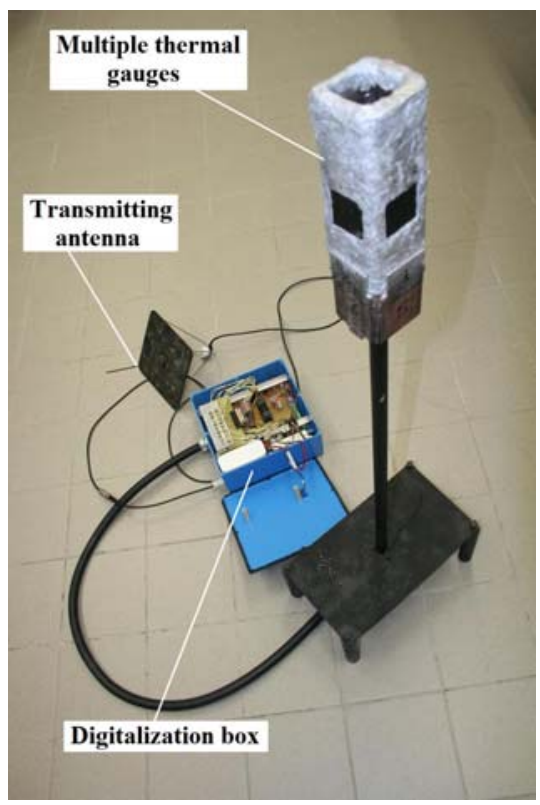

Figure 2. Photograph of the wireless heat flux sensor.

\begin{tabular}{|c|c|c|}
\hline$R_{e_{1}}$ & $\hat{R}$ & $C_{c o}$ \\
\hline 23.7 & 21.7 & 10.4 \\
\hline
\end{tabular}

Table 1. Values of the sensor parameters necessary to experimentally compute the total heat flux received by each gauge. 
As mentioned above, in our experiments the rear gauge (gauge 3) never sees the fire front, so it measures only the convective heat flux contribution. Consequently the radiative heat fluxes measured by the frontal and lateral gauges can be expressed as:

$$
\left\{\begin{array}{l}
\Phi_{i}^{\exp }(t)=C_{c o} \frac{d \bar{\theta}_{c o_{i}}}{d t}+\left(\frac{1}{\hat{R}}+\frac{1}{R_{e_{1}}}\right) \bar{\theta}_{c o_{i}} \\
\text { with } \Phi_{i}^{\exp }=\varphi_{i}^{\exp }-\varphi_{3}^{\exp } \text { and } \bar{\theta}_{c o_{i}}=\theta_{c o_{i}}-\theta_{c o_{3}}
\end{array}\right.
$$

\section{Image processing methodology}

The first step in the image processing technique used to obtain the forward or backward fire front positions was to acquire images of the fire with a video camera (30 images per second and a resolution of $640 \times 480$ pixels). Knowing the dimension of the burning area (the size of the combustion table), a Direct Linear Transformation was then performed in order to obtain real ground coordinates from pixel coordinates. This technique is used in a wide range of applications, including studies of fire behaviour [6]. The Ko et al. fire segmentation algorithm [13] was then applied on the images in order to eliminate non-fire pixels from the fire scene. Note that the choice of this algorithm for these experiments has been discussed in [14] using a supervised evaluation criterion. Lastly, the fire front lines positions can be deduced by taking the ground contour of the remaining fire pixels. This image processing technique can be summarized in the following four steps:

i) Cropping images of the propagation zone from a video recording.

ii) Direct Linear Transformation (DLT) to find real fire coordinates from camera fire pixel coordinates.

iii) Segmentation of the fire front lines.

iv) Computation of the fire front lines positions.

This segmentation algorithm [13] works in the RGB colour space with a Gaussian probability distribution model assuming the independence of the RGB channels for each pixel given by: 
$p_{i}(x, y)=\frac{1}{\sqrt{2 \pi \sigma_{i}}} \exp \left(\frac{\left(I_{i}(x, y)-\mu_{i}\right)^{2}}{2 \sigma_{i}^{2}}\right)$

In this expression $I_{i}(x, y)$ is the colour value for the $i^{\text {th }}$ colour channel in a fire image, $\mu_{i}$ and $\sigma_{i}$ are respectively the mean value and the standard deviation of $I_{i}$. To declare a pixel as a fire candidate, a threshold $\tau$ is used and the following condition is proposed:

$\left\{\begin{array}{l}p(I(x, y))=p_{R}\left(I_{R}(x, y)\right) \times p_{G}\left(I_{G}(x, y)\right) \times p_{B}\left(I_{B}(x, y)\right) \\ \text { if } p(I(x, y))>\tau \\ \text { then } I(x, y) \text { is a fire-pixel } \\ \text { else } I(x, y) \text { is not a fire-pixel }\end{array}\right.$

The final step in the image processing method was to calculate the fire front lines positions in order to produce accurate fire positions as input parameters for the thermal methodology. In the mathematical radiative flame model derived in section 2, the fire positions are determined only by their centred positions. Hence, only those positions are taken into account in the image processing approach. In order to compute the best estimation of the different fire front lines positions, a Savitzky-Golay smoothing filter [15] was applied to the fire front lines positions to eliminate the noise due to flame oscillations.

\section{Combining the two approaches}

Recently Rudz et al. [11] showed that the positions obtained after applying the image processing method are as reliable and accurate as those obtained after applying the heat flux approach. These authors highlighted the possibility of coupling the two methods to design a new metrological tool which would calculate average geometrical (fire front positions, tilt angle, flame length) and average physical (temperature, emissivity) parameters of the flame with a lower computational time than without coupling $[4,9]$. The main idea is to give fire front positions calculated by image processing as fixed parameters for the radiative heat flux approach in order to reduce the number of unknown parameters involved. 
Over the last two decades the inverse method used in engineering has been widely discussed by several authors $[16,17,18]$. The problem presented in this paper is one of parameter estimation because the flame model is known, but not the variables involved [19].

The inverse problem considered here can be stated as the following bound constrained minimization problem:

minimize $J(\xi)$

subject to $\xi_{l} \leq \xi \leq \xi_{u}$

with $J(\xi)=\frac{1}{\sum_{k=1,2,4}\left\|\Phi_{k}^{\exp }\right\|}\left(\left\|\Phi_{a}^{t h}(\xi)-\Phi_{1}^{\exp }\right\|+\left\|\Phi_{l}^{t h}(\xi)-\Phi_{2}^{\exp }\right\|+\left\|\Phi_{l}^{t h}(\xi)-\Phi_{4}^{\exp }\right\|\right)$

where $\xi=\left(y_{f_{1}}^{k}, y_{f_{2}}^{k}, \ldots, y_{f_{N}}^{k}, l_{f}, \phi_{f}\right)$ is the decision variables vector that will be identified, $\xi_{l}, \xi_{u}$ are respectively the lower and upper bounds of these variables, the vector $\left(y_{f_{1}}^{k}, y_{f_{2}}^{k}, \ldots, y_{f_{N}}^{k}\right)$ represents, for $k=B$, the backward or, for $k=F$, the forward fire front positions, and $\phi_{f}=K_{f} \frac{B T_{f}^{4}}{\pi}$ is the radiative heat flux of the flame per unit volume. $\|\cdot\|$ denotes the usual Euclidean norm, $\Phi_{a}^{\text {th }}, \Phi_{l}^{\text {th }}$ are the theoretical radiative heat fluxes given respectively by expressions (14) and (15) and $\Phi_{k}^{\exp } \forall k=1,2,4$ are the experimental radiative heat fluxes measured by the wireless multiple thermal gauges. Note that we should identify the two functions $y_{f}^{B}=y_{f}^{B}(t), y_{f}^{F}=y_{f}^{F}(t)$, but that in order to reduce the computational cost these functions have been considered at discrete time values.

A Generalized Pattern Search algorithm [20], provided by the "Genetic Algorithm and Direct Search” Toolbox of Matlab ${ }^{\circledR}$, is used to solve the bound constrained optimization problem of relation (20). The particularity of this optimization algorithm is that it does not require any information about the gradient of the objective function, whereas traditional optimization methods need gradient or higher derivatives information to search for an optimal point. In fact, this method is a global algorithm, named direct search method, which searches a set of points around the current point, looking for one where the value of the objective function is lower than the value at the current point. 


\section{Experimental conditions}

The fuel bed used for the experiments was a load of $1.2 \mathrm{~kg} \cdot \mathrm{m}^{-2}$ of straw placed on a combustion table. The table is made of cellular concrete and has an effective burning area of $2 \mathrm{~m}^{2}(2 \mathrm{~m} \times 1 \mathrm{~m})$ with a depth of $0.2 \mathrm{~m}$. The table can be inclined in order to simulate slope conditions. The different slopes simulated were $-10^{\circ}$, $0^{\circ}, 10^{\circ}$ and $20^{\circ}$. The complete experimental device also includes a wireless heat flux sensor and a video camera (see Figure 3 and Figure 4).

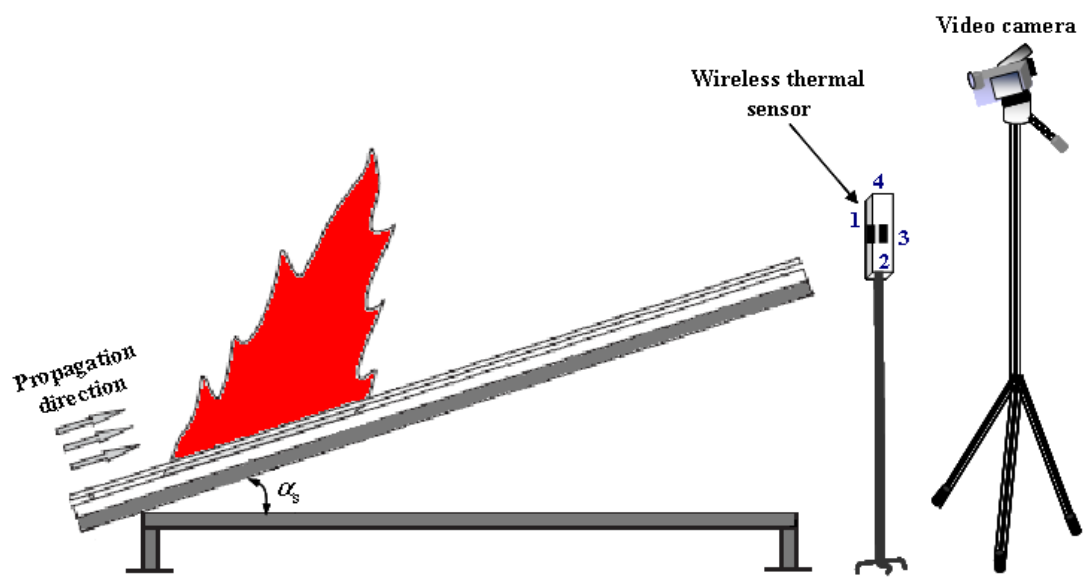

Figure 3. Schematic overview of the experimental device.

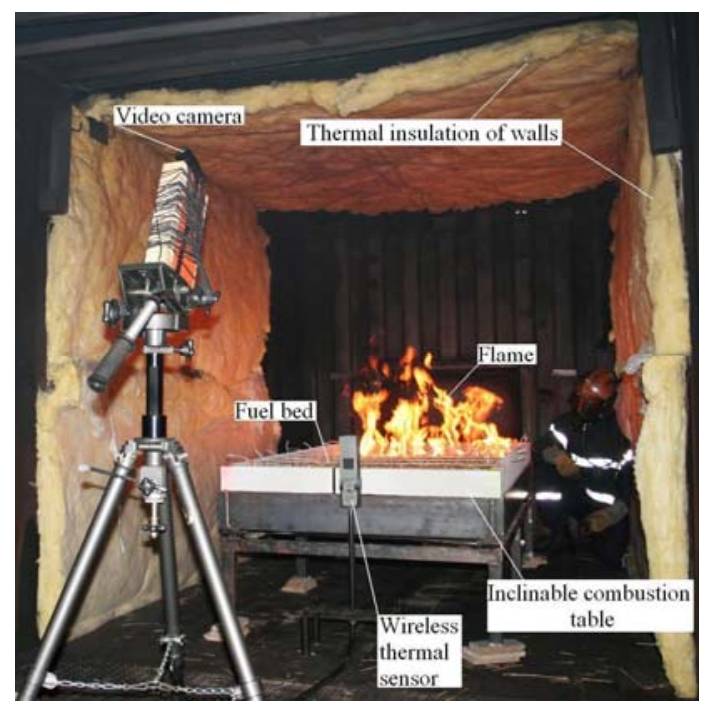

Figure 4. Photograph of the experimental device. 


\section{Results and discussion}

This section presents the results obtained for laboratory experimental fires after combining the two methodologies. Fire front positions were determined by image processing (see Figure 5) and then introduced as inputs in equation (20), the objective function which had to be minimized.

The calculation of flame characteristics by the inverse method gave the results presented in Table 2. It should be noted that the apparent flame temperature was computed according to equation (21) and that flame emissivity was obtained thanks to Beer's law [21], given by formula (22), after assuming an extinction coefficient of $0.72 \mathrm{~m}^{-1}$ experimentally determined for forest fuels by Àgueda et al. [22].

$T_{f}=\left(\frac{\phi_{f} \pi}{K_{f} B}\right)^{1 / 4}$

$\varepsilon_{f}=1-\exp \left(-K_{f} \delta_{f}\right)$

with $\delta_{f}$ the mean flame depth for each slope value. It should be pointed out that the values of $\varepsilon_{f}$ calculated by this relation and reported in this table are in the range of 0.11 to 0.66 for a flame depth from 0.27 to $1.88 \mathrm{~m}$ and 0.12 to 0.94 for a flame depth from 0.15 to $2 \mathrm{~m}$ given respectively by Beyreis et al. [23] and Hägglund and Persson [24]. The same range of emissivity values is also obtained by Dupuy et al. [25]. 


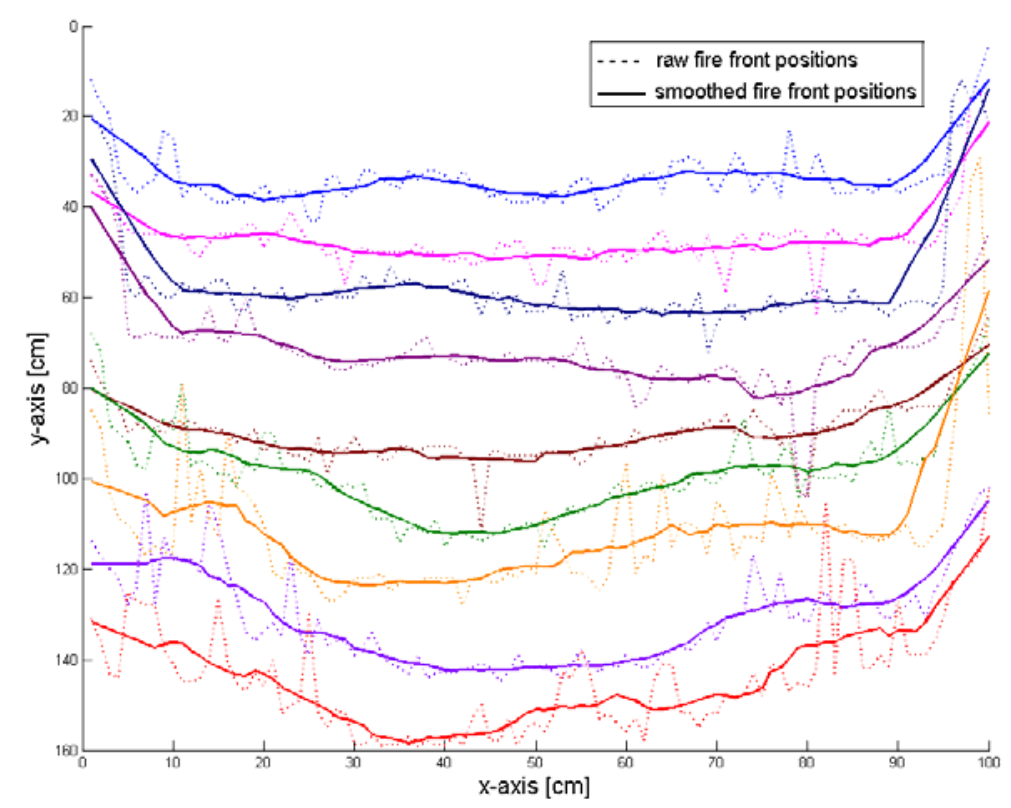

Figure 5. Example of fire forward front profile at different times for a fire spreading without slope.

\begin{tabular}{|l|l|l|l|l|}
\hline Slope $\left[^{\circ}\right]$ & -10 & 0 & +10 & +20 \\
\hline Flame length $l_{f}[\mathrm{~cm}]$ & 53.5 & 65.2 & 116.5 & 112.0 \\
\hline $\begin{array}{l}\text { Flame radiative heat flux } \\
\phi_{f}\left[\mathrm{~kW} \cdot \mathrm{m}^{-3}\right]\end{array}$ & 7.630 & 7.624 & 24.023 & 40.552 \\
\hline $\begin{array}{l}\text { Apparent flame temperature } \\
T_{f}[\mathrm{~K}]\end{array}$ & 875 & 875 & 1166 & 1329 \\
\hline Flame emissivity $\varepsilon_{f}$ & 0.18 & 0.18 & 0.25 & 0.27 \\
\hline Mean flame thickness $\delta_{f}[\mathrm{~cm}]$ & 28.2 & 27.0 & 40.8 & 42.9 \\
\hline
\end{tabular}

Table 2. Average values of radiative heat flux and flame length, temperature and emissivity for tests carried out under different slopes.

The different values of the fire front positions determined by this new metrological tool based on the coupling of two continuous methods (image processing and heat flux sensor) are plotted in Figure 6 for down and up-slopes. The rates of spread for these slope values are deduced by means of a linear regression using computed values of the fire front positions. The evolution of the rate of spread of the forward and backward fire fronts as a slope function is 
illustrated in Figure 7. This Figure shows that the rates of spread of the forward fire front are higher than those of the backward front and their values increase for different slope values due to flame radiation. The same trend has been observed by Morandini et al. [26] and Dupuy et al. [27] using respectively Pinus pinaster and Pinus halepensis needles as a fuel bed.

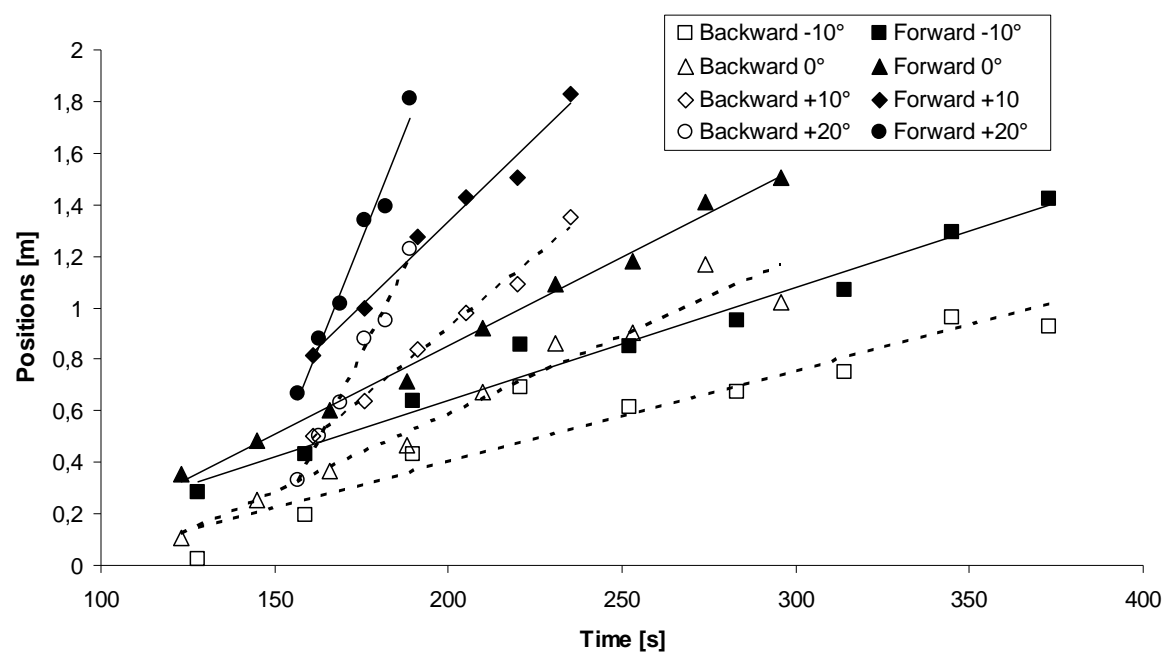

Figure 6. Centred forward and backward fire front positions versus time for different slopes.

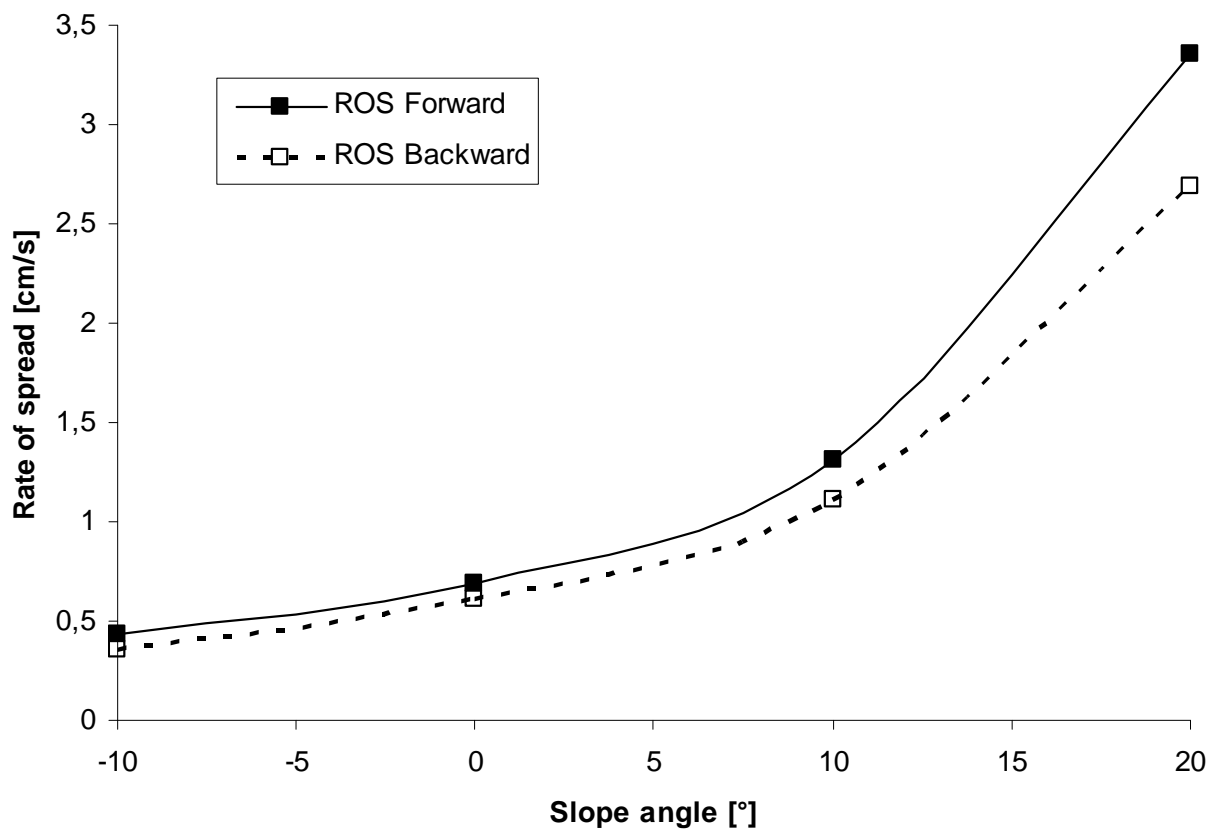

Figure 7. Computed rate of spread for down-slope and up-slope fires spread across straw bed. 


\section{Conclusion}

Several studies in the forest fire literature have used image processing to provide geometrical characteristics of the fire front. However, there has been little research into the determination of these characteristics with other physical parameters using the measurement of heat flux which is the only relevant physical quantity that is measurable.

In this paper, a flame model with mean thermal and radiative properties has been derived from the radiative transfer equation. The equations obtained give simple relations to minimize the differences, in terms of the usual Euclidean norm, between the theoretical and the experimentally measured radiative heat fluxes. It has been shown that coupling image processing and the wireless heat flux sensor gives results that are in good agreement with those found in the literature and can help to design a new robust metrological tool for forest fires. An important advantage is that the computational time of the new metrological tool proposed here is twice as fast as the one without implementation of the image processing method.

\section{References}

1. Viegas DX, Palheiro PM, Pita LP, Ribeiro LM, Cruz MG, Ollero A, Arrue B, Dios Ramiro M (2006) Analysis of fire behaviour in Mediterranean shrubs: The Gestosa fire experiments (Portugal). Forest Ecology and Management 234:S101.

2. Santoni PA, Simeoni A, Rossi JL, Bosseur F, Morandini F, Silvani X, Balbi JH, Cancellieri D, Rossi L (2006) Instrumentation of wildland fire: Characterisation of a fire spreading through a Mediterranean shrub. Fire Safety Journal 41:171-184.

3. Sullivan AL (2007) Convective Froude number and Byram's energy criterion of Australian experimental grassland fires. Proceedings of the Combustion Institute 31:2557-2564.

4. Chetehouna K, Séro-Guillaume O, Sochet I, Degiovanni A (2008) On the experimental determination of flame front positions and of propagation parameters for a fire. International Journal of Thermal Sciences 47:1148-1157.

5. Lloret J, Garcia M, Bri D, Sendra S (2009) A Wireless Sensor Network Deployment for Rural and Forest Fire Detection and Verification. Sensors 9:8722-8747.

6. Pastor E, Àgueda A, Andrade-Cetto J, Muñoz M, Pérez Y and Planas E (2006) Computing the rate of spread of linear flame fronts by thermal image processing. Fire Safety Journal 41:569-579. 
7. Martinez-de Dios JR, Arrue BC, Ollero A, Merino L, Gómez-Rodríguez F (2008) Computer vision techniques for forest fire perception. Image and Vision Computing 26:550-562.

8. Chetehouna K, Zarguili I, Séro-Guillaume O, Giroud F, Picard C (2008) On the two ways for the computing of the fire front positions and the rate of spread. Modelling, Monitoring and Management of Forest Fires, WIT Transactions on Ecology and the Environment 119:3-12.

9. Chetehouna K, Séro-Guillaume O, Bernardin D (2009) Detection of the Positions and Computing the Rate of Spread of Fire Fronts Using a Radiative Flame Model and Inverse Method. In: Eduards Gomez and Kristina Alvarez (ed) Forest Fires: Detection, Suppression, and Prevention. Nova Science Publishers, Inc, Chapter 8.

10. Séro-Guillaume O, Ramezani S, Margerit J, Calogine D (2008) On large Scale Forest Fires Propagation Models. International Journal of Thermal Sciences 47:680-694.

11. Rudz S, Chetehouna K, Séro-Guillaume O, Pastor E, Planas E (2009) Comparison of two methods for estimating fire positions and the rate of spread of linear flame fronts. Measurement Science and Technology 20:10pp.

12. Siegel R, Howell J (2002) Thermal radiation heat transfer. Taylor \& Francis (4 ${ }^{\text {th }}$. ed), New York.

13. Ko BC, Cheong KH, Nam JY (2009) Fire detection based on vision sensor and support vector machines. Fire Safety Journal 44:322-329.

14. Rudz S, Chetehouna K, Hafiane A, Séro-Guillaume O, Laurent H (2009) On the Evaluation of Segmentation Methods for Wildland Fire. In: J. Blanc-Talon et al. (ed), LNCS 5807:12-23.

15. Orfanidis SJ (1996) Introduction to Signal Processing, Prentice-Hall, Englewood Cliffs, NJ.

16. Huang $\mathrm{CH}$, Ozisik MN (1992) Inverse problem of determining unknown wall heat flux in laminar flow through a parallel plate duct. Numerical Heat Transfer, Part A 21:55-70.

17. Li HY, Yan WM (2000) Inverse convection problem for determining wall heat flux in annular duct flow. Journal of Heat Transfer 122:460-464.

18. Lee KH, Baek SW, Kim KW (2008) Inverse radiation analysis using repulsive particle swarm optimization algorithm. International Journal of Heat and Mass Transfer 51:27722783.

19. Beck JV, Blackwell B, Clair C St (1985) Inverse Heat Conduction, Ill-Posed Problems. John Wiley \& Sons, New York.

20. Lewis RM, Torczon V (1999) Pattern Search Algorithms for Bound Constrained Minimization. SIAM Journal on Optimization 9:1082-1099.

21. Monod B, Collin A, Parent G, Boulet P (2009) Infrared radiative properties of vegetation involved in forest fires. Fire Safety Journal 44:88-95.

22. Àgueda A, Pastor E, Pérez Y, Planas E (2010) Experimental study of the emissivity of flames resulting from the combustion of forest fuels. International Journal of Thermal Sciences 49:543-554. 
23. Beyreis JR, Monsen HW, Abbasi AF (1971) Properties of wood crib flames. Fire Technology 7:145-155.

24. Hägglund B, Persson LE (1974) An Experimental Study of the Radiation from Wood Flames. Försvarets Forskningsanstalt Huvudenhet, FoU-brand, Stockholm.

25. Dupuy JL, Vachet P, Maréchal J, Meléndez J, de Castro AJ (2007) Thermal infrared emission-transmission measurements in flames from a cylindrical forest fuel burner. International Journal of Wildland Fire 16:324-340.

26. Morandini F, Santoni PA, Balbi JH (2001) The contribution of radiant heat transfer to laboratory-scale fire spread under the influences of wind and slope. Fire Safety Journal 36:519-543.

27. Dupuy JL, Vachet P, Maréchal J (2006) Fuel bed temperature measurements in laboratory fires spreading over a slope. V International Conference on Forest Fire Research D.X. Viegas (ed). 